\title{
El consumo global del agua y la sustentabilidad
}
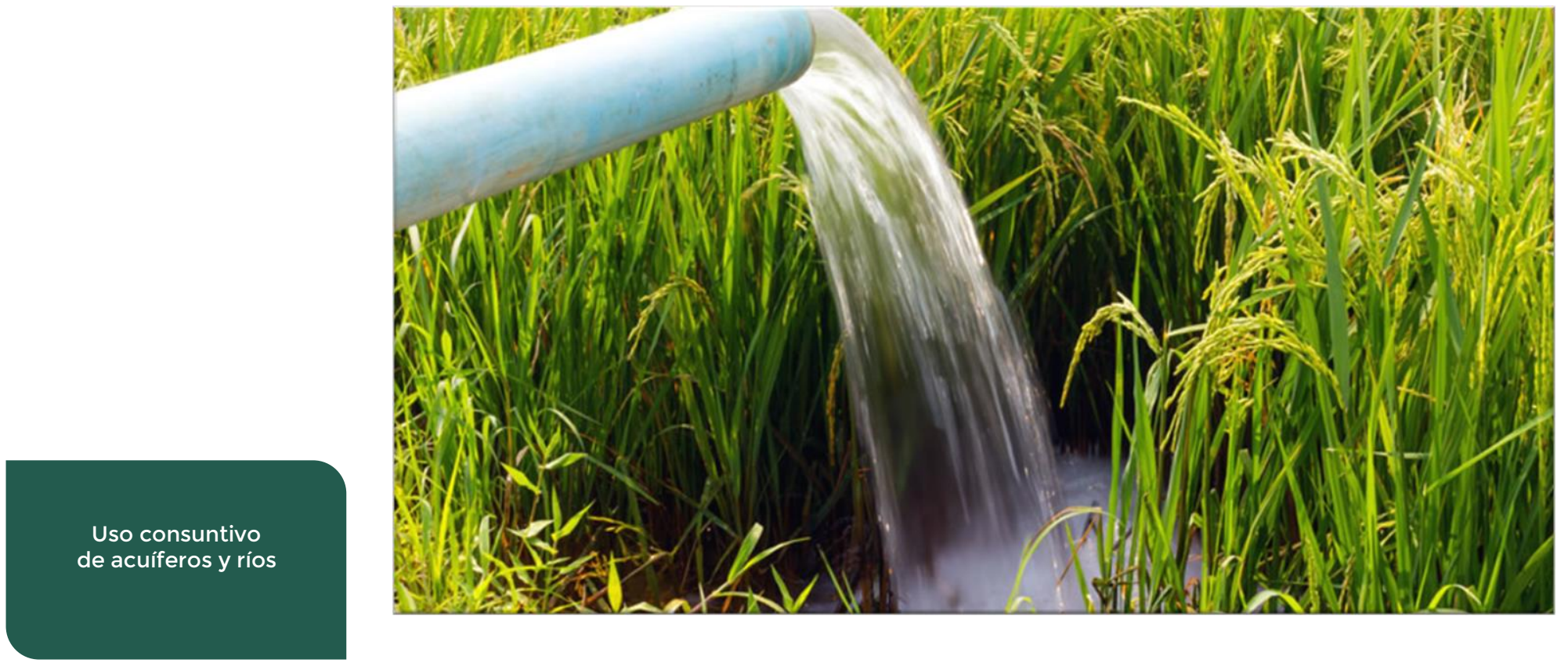

En muchos lugares de todos los continentes se ha registrado un uso consuntivo de acuíferos y ríos que excede por mucho los niveles deseados de sustentabilidad.

En años recientes se ha observado en la mayoría de los países un incremento acelerado, en términos absolutos y relativos, en el uso de agua subterránea. Solo en un pequeño número de naciones se ha logrado la estabilización -e incluso la recuperación- de los sistemas hídricos subterráneos. Wada et al. (2014) estiman que las extracciones de agua subterránea entre 1979 y 2010 crecieron de 610 mil millones a 1 billón 200 mil millones de $\mathrm{m}^{3}$ /año, lo que representa un incremento de $85 \%$, mientras que el porcentaje que representa el agua subterránea de las extracciones totales incrementó de 32.5 a $36.4 \%$ en ese mismo periodo. Durante el periodo de 1979 a 1990, la extracción global de agua subterránea se incrementó en el orden de $1 \%$ al año; sin embargo, de forma notable, en el periodo de 1990 a 2010 , caracterizado por un modelo de desarrollo extractivista, el incremento anual se triplicó, lo que dio como resultado un incremento anual en las extracciones del tres por ciento.

Esto indica una creciente presión sobre el agua subterránea a nivel global, que se explica por la lógica de un modelo que contempla al agua como un recurso a ser explotado y que, en el caso del agua subterránea, la convierte en un fusible ante la escasez de agua superficial y la reducción en la 
construcción de presas de almacenamiento a nivel global. En Europa, las extracciones de agua subterránea corresponden al 30 \% del total de extracciones, y estas no se han visto incrementadas de forma sustancial en las últimas décadas; caso contrario a lo que ocurre en Norteamérica y Centroamérica, donde las extracciones de agua subterránea se han incrementado en más del 40 \% en el periodo de 1979 a 2010, alcanzando un valor relativo del $60 \%$ respecto a las extracciones totales. En el oeste asiático, la extracción de agua subterránea se triplicó, y representaba el 70 \% de las extracciones de agua en 2010. En el sur y este de Asia, las extracciones de agua subterránea casi se duplicaron en ese mismo periodo, mientras que, en el norte de África, las extracciones de agua subterránea representan casi $30 \%$ del total. En otras regiones del mundo abundantes en ríos, como son el sureste asiático o Sudamérica, las extracciones de agua subterránea para diversos usos representan menos del $20 \%$ del total.

Gleeson et al. (2012) reportan que, a nivel global, las extracciones de agua subterránea exceden la disponibilidad de los acuíferos en un $20 \%$, definiendo la disponibilidad de agua subterránea como como el valor de recarga menos la contribución del agua subterránea a los flujos ambientales o gasto base. Por si esto fuera poco, cerca de 1,700 millones de personas en el mundo vive en regiones donde las extracciones exceden la disponibilidad, lo que pone en riesgo no solamente su seguridad hídrica, sino la salud de los ecosistemas. Según este estudio, los lugares con los mayores niveles de sobreexplotación de acuíferos son: India, Pakistán, Arabia Saudita, Irán, México, Estados Unidos de América, el norte de África, China y Europa del este. En los acuíferos correspondientes a la cuenca alta del Ganges y el bajo Indo, en la frontera de India y Pakistán, los valores relativos de las extracciones de agua subterránea respecto a la disponibilidad promedian 54 y 18, respectivamente. En los acuíferos del norte y sur de Arabia Saudita, estos valores son de 48 y 39, respectivamente, mientras que en el los acuíferos del Medio Oriente, en Siria e Irán, al sur del mar Caspio, estos valores son de 20 y 98 , respectivamente. En los acuíferos de las Altas Llanuras y del Valle Central en los Estados Unidos, estos valores rondan 9.0 y 6.4. En Egipto, el acuífero del delta del río Nilo da un valor de 32. En los acuíferos de la llanura y del norte de China estos valores son de 7.9 y 4.5. En el caso del acuífero de la cuenca del Danubio, debajo de partes de Hungría, Austria y Rumania, esta proporción es de 7.4. Por último, en el caso de México, para los acuíferos del oeste y centro el país, se reportan valores de 27 y 9.1, respectivamente.

En un estudio anterior, Wada et al. (2012) encontraron que, en el año 2000, las extracciones de agua subterránea no renovable constituyeron cerca del $20 \%$ del total de agua utilizada para riego, reportando las mayores extracciones de agua subterránea no renovable para uso agrícola en India, Pakistán, Estados Unidos de América, Irán, China, México y Arabia Saudita. Más aún, encontraron que, globalmente, las extracciones de agua subterránea no renovable se triplicaron en el periodo de tiempo definido entre los años 1960 y 2000.

Adicionalmente, Hoekstra y Mekonnen (2012) comprobaron que algunos de los países con los mayores niveles de sobreexplotación de agua subterránea se encuentran también entre los más grandes exportadores; es decir, entre los países que utilizan agua subterránea para producir alimentos de exportación. Estos son: Estados Unidos, China, India y Pakistán. De hecho, India ha sido reconocido como el exportador de agua virtual más grande del mundo, mientras que Estados Unidos se encuentra en tercer sitio. Análisis posteriores han demostrado que incluso en países como Egipto e Irán, con balanza positiva en agua virtual (valor que compara el volumen de agua utilizada para exportaciones respecto a aquella recibida en importaciones), el valor del agua virtual de las exportaciones es también sustancial (Karandish y Hoekstra, 2017; Aabdelkader et al., 2018).

Cabe señalar que las estimaciones de extracciones de agua subterránea y las tasas de recarga de los acuíferos son variables que contienen un cierto porcentaje de incertidumbre, y la única forma de 
reducirla es a través de la medición adecuada de ambas. De acuerdo con Margat y Van der Gun (2013), en el año 2010, las extracciones totales de agua subterránea rondaron los 982 mil millones de $\mathrm{m}^{3} /$ año. Reportaron también que la agricultura es responsable del 70 \% de las extracciones globales de agua subterránea, mientras que el suministro de agua potable es responsable del 20 \% y la industria del nueve por ciento.

Wada et al. (2014) proveen una amplia reseña de diferentes estimaciones de extracciones de agua subterránea que resultan de un conjunto de modelos globales, que varían de entre 545 mil millones $\mathrm{m}^{3}$ /año (Siebert et al. 2010; solo considerando agua subterránea para uso agrícola) hasta 1 billón 700 mil millones de $\mathrm{m}^{3}$ /año (Wiseer et al. 2010). La debilidad de las estimaciones indirectas, que tienen su base en estadísticas nacionales, es que dependen de valores cuya confiabilidad y exactitud es completamente desconocida, mientras que las estimaciones de modelos contienen incertidumbre epistémica asociada a las hipótesis de los mismos y a los datos de entrada que se utilizan para su calibración y ejecución.

En este sentido, es importante hacer notar que los valores de extracción de agua subterránea deben ser considerados con un alto margen de error. Lo mismo se sostiene para la estimación de las tasas de recarga. De acuerdo con FAO et al. (2016), cuyo estudio se basó en valores provenientes de estadísticas nacionales, las extracciones globales de agua subterránea equivalen al 8 \% de la media global de recarga. Este valor global evidentemente esconde, por la escala, las muy grandes diferencias regionales que se mostraron en los estudios de Gleeson et al. (2012) o Margat y Van der Gun (2013). A pesar de la incertidumbre asociada a la estimación de extracciones de agua subterránea en muchos acuíferos, es claro, por los reportes de las personas que habitan estos territorios, que los niveles piezométricos se están abatiendo drásticamente.

Cuando consideramos el consumo de agua azul total en ríos y la comparamos con su disponibilidad, obtenemos una imagen igualmente desalentadora. Mekonnen y Hoekstra (2016) realizaron un estudio global a alta resolución espacial, encontrando que en el año 2000, dos tercios de la población mundial ( 4 mil millones de personas en ese momento) vivían en áreas con escasez hídrica severa al menos un mes al año; casi la mitad de esas personas con residencia en China e India. El número de personas que habitan en áreas con escasez hídrica severa entre 4 y 6 meses al año fue de 1,800 millones a 2,900 millones, mientras que 500 millones de personas viven en lugares con escasez severa de agua durante todo el año. En ese estudio, la disponibilidad de agua azul se calculó como el escurrimiento menos las necesidades del medio ambiente, definidas por los flujos ambientales (que se supone son del $80 \%$ del escurrimiento natural).

Se han definido cuatro niveles de escasez hídrica: baja, moderada, alta y severa.

La escasez baja indica que la huella hídrica azul (consumos) no excede la disponibilidad. La huella hídrica azul es, por tanto, menor del $100 \%$ de la disponibilidad de agua (menor al $20 \%$ del escurrimiento natural). Suponiendo que los requerimientos ambientales no son invadidos, este representa el nivel de condiciones de sustentabilidad en una región.

La escasez moderada se refiere a que la huella hídrica azul está entre 100 y 150 \% de la disponibilidad (entre 20 y $30 \%$ del escurrimiento natural) y donde se presume que los flujos ambientales no son considerados.

La escasez hídrica alta se da cuando la huella hídrica azul está entre 150 y $200 \%$ de la disponibilidad (entre 30 y $40 \%$ del escurrimiento natural). 
PERSPECTIVAS IMTA (0)

$\mathrm{N}^{\circ} .14,2020$

Por último, se determina escasez hídrica severa cuando la huella hídrica azul es mayor de $200 \%$ de la disponibilidad (mayor a $40 \%$ del escurrimiento natural).

En un estudio anterior, Hoekstra et al. (2012) mostraron que la huella hídrica azul era no sustentable al menos un mes al año en el 55 \% de las 405 cuencas más grandes del mundo. Las cuencas analizadas de forma conjunta comprenden el 69 \%del escurrimiento superficial global, 75 \% del área global bajo riego y 65 \% de la población mundial. La severidad y duración de la escasez son muy importantes cuando se consideran los impactos sociales, económicos y ambientales asociados a este fenómeno. Por ejemplo, de este estudio se desprende que la cuenca más poblada que enfrenta una escasez hídrica severa durante todo el año se ubica en el norte de China (la cuenca de Yongding He), con una superficie de 214,000 km2 y una densidad de población de 425 personas por kilómetro cuadrado.

Estos estudios globales, acompañados de la evidencia indirecta registrada por los conflictos entre usuarios de agua a nivel local en zonas con una reconocida escasez, nos señalan la necesidad urgente de regionalizar las conclusiones para las cuencas de nuestro país. En el IMTA estamos ciertos de que es posible avanzar a un México sin desigualdad, con desarrollo económico y con calidad ambiental de la mano del uso sustentable del agua. Pero para ello, requerimos utilizar la evidencia científica moderna, el pensamiento crítico, la ética y la transparencia de la información, que nos permitan construir una solución con la sociedad.

\section{Referencias:}

Aabdelkader A, Elshorbagy A, Tuninetti M, Laio F, Ridolfi L, Fahmy H, Hoekstra AY, (2018) National water, food and trade modeling framework: The case of Egypt. Science of the Total Environment, 639: 485-496.

FAO, UNESCO, IAH, World Bank Group, GEF (2016) Global diagnostic on groundwater governance, Food and Agriculture Organization, Rome, Italy.

Gleeson T, Wada Y, Bierkens MFP, van Beek LPH (2012) Water balance of global aquifers revealed by groundwater footprint. Nature, 488 (7410): 197-200.

Hoekstra AY, Mekonnen, MM, Chapagain, AK, Mathews RE, Richter BD (2012) Global monthly water scarcity: Blue water footprints versus blue water availability. PLoS ONE, 7(2): e32688.

Hoekstra y Mekonnen (2012) The waterfootprint of humanity. Proceedings of the National Academy of Sciences, 109(9): 3232-3237.

Karandish MM, Hoekstra, AY (2017) Informing national food and water security policy through water footprint assessment: The case of Iran. Water 9(11): 831.

Margat y Van der Gun (2013) Groundwater around the world: A geographic synopsis, CRC Press, Leiden, the Netherlands.

Mekonnen y Hoekstra (2016) Four billion people facing severe water scarcity. Science Advances, 2(2): e1500323.

Siebert S, Burke J, Faures JM, Frenken K, Hoogeveen J, Döll P, Portmann FT (2010) Groundwater use for irrigation: A global inventory, Hydrology and Earth System Sciences, 14: 1863-1880

Wada Y, Van Beek LPH, Bierkens, MFP. (2012) Nonsustainable groundwater sustaining irrigation: A global assessment. Water Resources Research, 48: WOOL06.

Wada Y, Wisser D, Bierkens, MFP (2014) Global modeling of withdrawal, allocation and consumptive use of surface water and groundwater resources. Earth System Dynamics, 5: 15-40.

Wiseer D, Fekete, NM, Vörösmaty, CJ, Schumann, AH. (2010) Reconstructing 20th century global hydrography: A contribution to the global Terrestrial Network-Hydrology (GTN-H). Hydrology and Earth System Sciences, 14(1): 1-24. 